\title{
Einleitende Worte zur Bewertung des Zusammenwirkens von Mensch und Maschine
}

\section{Susanne Beck}

\section{Zusammenfassung}

Die zunehmende Bedeutung von Künstlicher Intelligenz, (KI) - der Begriff wird hier nicht als Analogie zu menschlicher Intelligenz verwendet, sondern als anerkannte Bezeichnung für im weitesten Sinne autonome, selbstlernende technische Systeme - stellt die Gesellschaft und damit auch das Recht vor erhebliche, neuartige Herausforderungen. Menschliche Entscheidungen, die durch KI vorbereitet oder gar ersetzt werden, können nicht in derselben Weise ethisch und rechtlich bewertet werden wie traditionelle, durch technische Werkzeuge unterstützte Entscheidungen. Die Besonderheiten dieser neuartigen Form des Zusammenwirkens menschlicher und künstlicher Intelligenz in verschiedenen Lebensbereichen sind in den ersten beiden Teilen dieses Bandes ebenso detailliert wie innovativ aufgezeigt.

Die zunehmende Bedeutung von Künstlicher Intelligenz (KI) - der Begriff wird hier nicht als Analogie zu menschlicher Intelligenz verwendet, sondern als anerkannte Bezeichnung für im weitesten Sinne autonome, selbstlernende technische Systeme - stellt die Gesellschaft und damit auch das Recht vor erhebliche, neuartige Herausforderungen. Menschliche Entscheidungen, die durch KI vorbereitet oder gar ersetzt werden, können nicht in derselben Weise ethisch und rechtlich bewertet werden wie traditionelle, durch technische Werkzeuge unterstützte Entscheidungen. Die Besonderheiten dieser neuartigen Form des

\section{S. Beck $(\bowtie)$}

Kriminalwissenschaftliches Institut der Leibniz Universität, Hannover, Deutschland E-Mail: susanne.beck@jura.uni-hannover.de 
Zusammenwirkens menschlicher und künstlicher Intelligenz in verschiedenen Lebensbereichen sind in den ersten beiden Teilen dieses Bandes ebenso detailliert wie innovativ aufgezeigt.

Ein gelungenes Zusammenwirken dieser neuen Technologien mit dem Menschen bedarf einer moralischen und rechtlichen Einhegung. Unser derzeitiges Gesellschaftssystem basiert auf der Idee, dass menschliche Entscheidungen und Handlungen durch moralische und rechtliche Regeln im Vorhinein gesteuert und im Nachhinein bewertet werden können. $\mathrm{Zu}$ den eher präventiven Vorgaben gehören etwa Genehmigungen, die vor einer bestimmten Handlung eingeholt werden müssen (Baugenehmigung, Genehmigung für die Erprobung von Medizinprodukten, etc.), zwischen Parteien vereinbarte Vertragsbedingungen oder Verhaltensrichtlinien bestimmter Gruppierungen (etwa der Ärztekammern). $\mathrm{Zu}$ den eher bewertenden bzw. ein ungewolltes Ergebnis korrigierenden Regelungen gehören beispielsweise Vertragsstrafen, die zivilrechtliche Haftung für Schädigungen, sowie die Tatbestände des Strafgesetzbuchs.

Die der Moral und dem Recht zugrunde liegende Idee der Regulierung menschlichen Verhaltens basiert grundsätzlich auf dem Gedanken der Verantwortung des Menschen für sein Tun. Diese gegenseitige Zuschreibung von Verantwortung ist nicht nur grundlegend für unser zwischenmenschliches Miteinander, sondern auch für unser Selbstbild als Wesen mit einem Freien Willen und Gestaltungshoheit über unser Leben.

Von der umfassenden Verantwortung für ein Geschehen gibt es schon in unserem gängigen System Ausnahmen, etwa für Minderjährige oder psychisch Erkrankte, aber auch für bestimmte Situationen, in denen der Handelnde für ein Ereignis zumindest nicht umfassend verantwortlich gemacht werden kann. Ein Beispiel hierfür ist die Eingebundenheit in ein kollektives System, z. B. in einen Unrechtsstaat oder eine kriminelle Organisation, aber auch ganz generell die Kooperation mit anderen Menschen, die dazu führt, dass jeder nur einen Teilbeitrag zu einem bestimmten Ergebnis leistet. Diese Ausnahmen spielen, wie wir sogleich sehen werden, für das Zusammenwirken mit KI durchaus eine Rolle. Es ist aber eben immer auch zu bedenken, dass eine zu weitgehende Reduktion von individueller Verantwortung zugleich unser gesellschaftliches System und unser auf Zuschreibung beruhendes Selbstbild verunsichern kann.

Betrachten wir aber nun, was genau an der aktuellen technologischen Entwicklung die traditionelle Bewertung derart erschwert. Wie die Beiträge dieses Bandes deutlich zeigen, verändert sich der Prozess einer Entscheidung durch die neuen Systeme. Das ist jedenfalls dann deutlich erkennbar, wenn die Maschine die Entscheidung alleine, also autonom, trifft. Hier haben zwar Menschen die Maschine programmiert und sich für die Nutzung der Maschine entschieden. 
Das allein kann jedoch keine umfassende Verantwortung für die Entscheidung der Maschine in der konkreten Situation bedeuten. Es ist nahezu gerade Sinn und Zweck der Übertragung von Entscheidungen auf Maschinen, uns von einer umfassenden Kontrolle zu entlasten, uns nicht alle Informationen selbst einzuholen und die Situation nicht selbst in allen Details einschätzen zu müssen. Gerade wenn die Maschine aus verschiedenen Prozessen selbst dazu lernt, ist weder im Vorhinein einschätzbar, wie sie sich entscheiden wird, noch im Nachhinein beweisbar, welche Vorgaben zu welcher Entscheidung führten. Hier noch von einer umfassenden Verantwortung der beteiligten Menschen zu sprechen, dehnt das Konzept Verantwortung zu weit aus.

Das gilt meines Erachtens aber sogar für Situationen, in denen die Maschine die Entscheidung vorbereitet, da der Mensch durch diese Vorbereitung einen stark verringerten Entscheidungsspielraum hat (etwa aufgrund einer besonders hohen psychischen Hemmschwelle, sich gegen die Maschine zu entscheiden, einer geringen Zeitspanne für die menschliche Reaktion oder fehlende Transparenz der maschinellen Vorschläge und daher die fehlende Möglichkeit, die Gründe der Maschine nachzuvollziehen). Es handelt sich also häufig um Konstellationen, in denen der oft geforderte Human in the Loop letztlich nur noch eine symbolische Funktion hat. Insofern gilt, dass die Gesellschaft vom Individuum jedenfalls nicht mehr erwarten kann, als ihm möglich ist. Bei der Kooperation von Menschen mit Robotern und KI-gestützten Systemen ist also im Einzelfall genau zu prüfen, wie sich die Einbeziehung der Maschine auf den Entscheidungs- und Handlungsspielraum des Menschen auswirkt und ob das seine Verantwortung ausschließt oder zumindest maßgeblich verringert.

Diese Überlegungen haben also einerseits zur Folge, dass wir unsere gängigen Verantwortungskonzepte überdenken sollten. Dabei sind die bestehenden Überlegungen wie etwa die zur kollektiven Verantwortung oder die bereits erwähnten existierenden Ausnahmen hilfreich. Zugleich können sie aber die aktuellen Entwicklungen in der KI nicht immer umfassend einbeziehen. Insbesondere besteht die Gefahr, dass der Nutzer der Maschine, also derjenige, der die von ihr vorgeschlagene Entscheidung absegnet bzw. die Überwachung übernehmen soll (etwa bei selbstfahrenden Kfz.), als eine Art Haftungsknecht verantwortlich gemacht wird, nur damit wir unsere bestehenden Verantwortungskonzepte auf diese neuen Entwicklungen anwenden können. Das wäre eine ungerechtfertigte Benachteiligung eines Einzelnen für eine Technologie, für die wir uns als Gesamtgesellschaft entscheiden. Wenn wir diese Entscheidung treffen, d. h. vermehrt künstliche Intelligenz in unsere Entscheidungen einbeziehen wollen, sollten wir auch die Konsequenzen tragen. 
Dass es trotzdem nicht sinnvoll ist, in all diesen Bereichen auf individuelle Verantwortung zu verzichten, zeigt sich an den oben erwähnten Herausforderungen, die das für die Gesellschaft und unser Selbstbild haben könnte. Deshalb ist es wichtig, dass wir das Zusammenwirken von menschlicher und künstlicher Intelligenz in einer Art und Weise gestalten, dass wir in bestimmten Kontexten und Entscheidungen doch noch eine Zurechnung von Verantwortung vornehmen können. Als erster Schritt ist dafür die Entscheidung zu treffen, in welchen Kontexten wir diese Zurechnung brauchen und in welchen wir darauf verzichten können - darüber sollten wir als Gesellschaft offen diskutieren. In einem zweiten Schritt ist dort, wo diese Zurechnung uns erforderlich erscheint, eine sogenannte Meaningful Human Control (MHC) über die künstliche Intelligenz zu garantieren. Dieser aus der Debatte um Autonome Waffensysteme stammende Begriff meint, dass der Mensch nicht nur eine scheinbare, sondern eine wirkliche Kooperation mit der Maschine durchführt, bei der er eine bedeutsame Kontrolle behält. Das kann je nach Lebensbereich ganz unterschiedlich ausgestaltet sein. So ist denkbar, dass die Maschine Gründe für ihren Vorschlag angeben muss. Oder die Maschine macht nicht nur einen Vorschlag, sondern mehrere nicht hierarchisierte Vorschläge, aus denen der Mensch auswählen muss. Oder aber der Mensch macht den Vorschlag, die Maschine berät ihn anschließend dazu. Eine besondere Herausforderung stellt Meaningful Human Control in Situationen dar, in denen nicht viel Zeit für die Entscheidung bleibt, etwa im Straßenverkehr, in kriegerischen Auseinandersetzungen oder im OP-Saal. Aber auch insofern finden bereits Debatten darüber statt, wie der Mensch die Kontrolle behalten könnte. Diese Debatten sind meines Erachtens der Öffentlichkeit in stärkerem Maß als bisher bekannt zu machen und sie ist dann in diese Entscheidungen einzubeziehen. Denn wann Verantwortung zuschreibbar ist, ist eben auch immer eine gesellschaftliche Frage - und hier ist durchaus denkbar, dass neue Gesellschaftsverträge über die Rolle von Mensch und Maschine erforderlich werden.

Dass unsere Zukunft von einem verstärkten Zusammenwirken zwischen menschlicher und künstlicher Intelligenz geprägt sein wird, lässt sich wohl kaum noch bestreiten oder bekämpfen. Dieses Zusammenwirken wird zudem gerade in unserer schnellen Welt mit ihren kaum noch beherrschbaren Datenmengen viele Vorteile mit sich bringen. Wir müssen meines Erachtens jedoch von Beginn an sicherstellen, dass wir Menschen über die Entwicklung insgesamt als auch über die einzelnen Entscheidungen im Rahmen dieses Zusammenwirkens eine hinreichende, inhaltlich bedeutsame Kontrolle behalten. 
Open Access Dieses Kapitel wird unter der Creative Commons Namensnennung 4.0 International Lizenz (http://creativecommons.org/licenses/by/4.0/deed.de) veröffentlicht, welche die Nutzung, Vervielfältigung, Bearbeitung, Verbreitung und Wiedergabe in jeglichem Medium und Format erlaubt, sofern Sie den/die ursprünglichen Autor(en) und die Quelle ordnungsgemäß nennen, einen Link zur Creative Commons Lizenz beifügen und angeben, ob Änderungen vorgenommen wurden.

Die in diesem Kapitel enthaltenen Bilder und sonstiges Drittmaterial unterliegen ebenfalls der genannten Creative Commons Lizenz, sofern sich aus der Abbildungslegende nichts anderes ergibt. Sofern das betreffende Material nicht unter der genannten Creative Commons Lizenz steht und die betreffende Handlung nicht nach gesetzlichen Vorschriften erlaubt ist, ist für die oben aufgeführten Weiterverwendungen des Materials die Einwilligung des jeweiligen Rechteinhabers einzuholen.

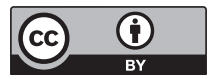

